\title{
Intraoperative Reparation of Superior Sagittal Sinus (SSS) Rupture with Autologous Fibrin Glue: Management of a Complication. Our Experience and Technical Note
}

\author{
Antonella Giugno, Rosario Maugeri, Francesca Graziano and Domenico G. lacopino
}

${ }^{1}$ Department of Experimental Biomedicine and Clinical Neurosciences, School of Medicine, Neurosurgical Clinic, University of Palermo, Via del Vespro 129, 90100, Palermo, Italy

\begin{abstract}
Management of complications in case of rupture of the superior sagittal sinus (SSS), during parasagittal meningiomas surgery represents a critical issue for neurosurgeons. Various techniques have been proposed to repair the sinus since the first reports. Nowadays, SSS reparation is achieved with haemostatic tools as Gelfoam or Surgicel, ligature, and through mechanic compression with cottonoids. Our paper is the first report of reparation of the SSS with an autologous fibrin glue system (Vivostat $\left.{ }^{\circledR}\right)$. We also describe our experience and the management of a common complication in the surgical treatment of lesions involving the sinus.
\end{abstract}

\section{Introduction}

Surgical treatment of lesions involving the Superior Sagittal Sinus (SSS) is a critical topic of discussion for neurosurgeons nowadays. In fact, there is an open debate on the management of parasagittal meningiomas, depending on the specific part of the sinus affected and on its grade of tumoral invasion. These lesions present important surgical challenges and several different techniques are used to repair the sinus and guarantee the hemostasis depending on the operators. The surgical management is highly subjective, especially in selected complicated cases.

Usually SSS reparation is achieved with hemostatic tools like Gelfoam or Surgicel, and through mechanic compression with cottonoids. Sometimes these devices are not sufficient. In this paper we report our experience with an autologous fibrin glue system (Vivostat ${ }^{\circ}$ ) which was used to repair the SSS in a patient with recurrent right fronto-parietal meningioma.

\section{Case Report}

The patient had previously undergone subtotal removal of right fronto-parietal parasagittal meningioma; grade III according to Sindou's classification. A small residual fragment involving the sinus remained. The patient underwent neuroradiological and clinical postoperative follow up which was free of symptoms. One year later brain MRI documented meningioma recurrence with volume increasing and further SSS invasion (grade IV) (Figure 1). The patient presented several epileptic seizures episodes and a mild but increasing left leg weakness. For this reason, a surgical procedure was performed to remove the tumor radically. Another fronto-parietal craniotomy

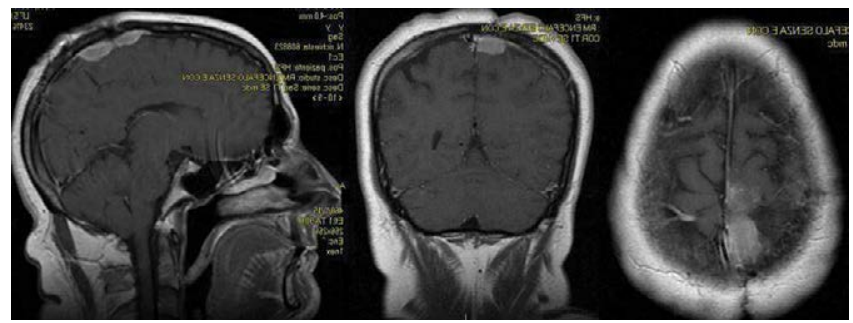

Figure 1: One year post. Op MR (T1 with gadolinium contrast agent, axial corneal and sagittal plane) that documents meningioma recurrence and SSS invasion (gradelV) extending about $2 \mathrm{~cm}$ away from the midline was performed, on the basis of the previous one. The lesion was removed in small fragments, partly with the aid of the ultrasound aspirator. The tumor showed a clear cleavage plane from the surrounding cerebral parenchyma with one fragment tenaciously adherent to the SSS (Figure 2A). During the surgical resection, a sudden profuse venous bleeding occurred from the sinus which was teared, (Figure 2B). At first, we apposed compression with cottonoids and used classical haemostatic devices as oxidized cellulose gauze; Gelfoam and Surgicel. Since profuse bleeding continued, we injected the autologous fibrin glue with the Vivostat ${ }^{\circ}$ system, (Figure 2C) easily achieving perfect haemostasis in a relatively small amount of time, and in a definitive, secure manner (Figure 2D). Finally, the resection was radical, without injuries to parenchymal or other vascular structures. We accomplished haemostasis of the residual cavity with fibrin glue once again, and a synthetic non-suturable dural patch was positioned to repair meningeal deficit. The outer surface of the dural patch was also coated with fibrin glue because of its sealing function. The patient had an excellent clinical outcome without any new neurological impairment. Post-operative brain angio-MR showed the complete patency of the SSS (Figure 3).

Hystopathology revealed a meningeal tumor with high cellularity, necrosis, Ki6: $10 \%$ and positivity for EMA and vimentin with a final diagnosis of a grade II atypical meningioma.

\section{Discussion}

In the last few years fibrin glues have gained growing importance as dural sealant devices, to prevent and/or repair cerebrospinal fluid

*Corresponding author: Domenico G. Iacopino, Universita degli Studi di Palermo, Experimental Biomedicine and Clinical Neurosciences, Via del vespro 129, Palermo, PA 90127, Italy, Tel: +39.328.0410463; Fax: +39.0916552393; E-mail: gerardo.iacopino@unipa.it

Received December 29, 2014; Accepted March 22, 2015; Published March 24 2015

Citation: GiugnoA, MaugeriR, GrazianoF,lacopinoDG(2015)IntraoperativeReparation of Superior Sagittal Sinus (SSS) Rupture with Autologous Fibrin Glue: Management of a Complication. Our Experience and Technical Note. J Neurol Disord 3: 222. doi: 10.4172/2329-6895.1000222

Copyright: (c) 2015 Giugno A, et al., This is an open-access article distributed unde the terms of the Creative Commons Attribution License, which permits unrestricted use, distribution, and reproduction in any medium, provided the original author and source are credited. 

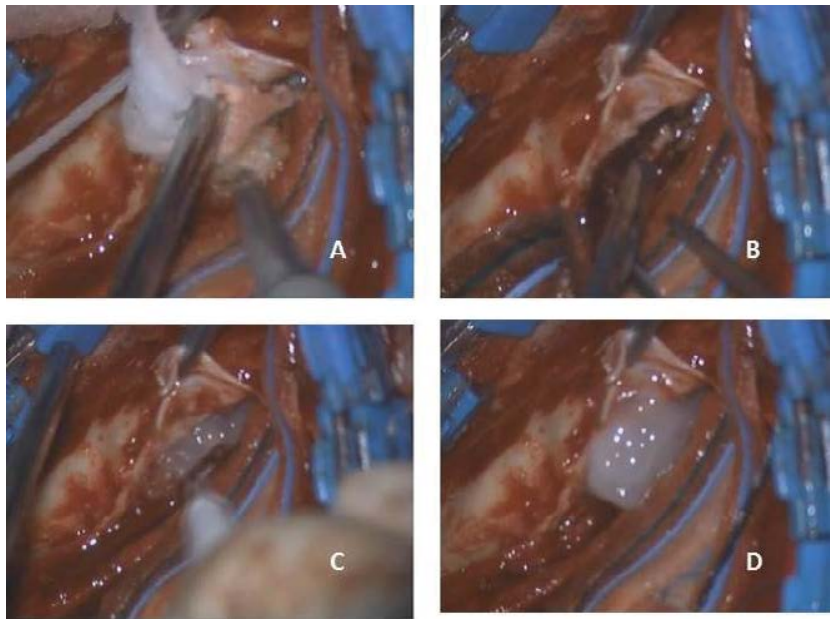

Figure 2: Intraoperative rupture of the SSS following removal of meningioma $(\mathrm{A}-\mathrm{B})$, autologous fibrin glue that permits hemostasis without occluding the SS (C-D).

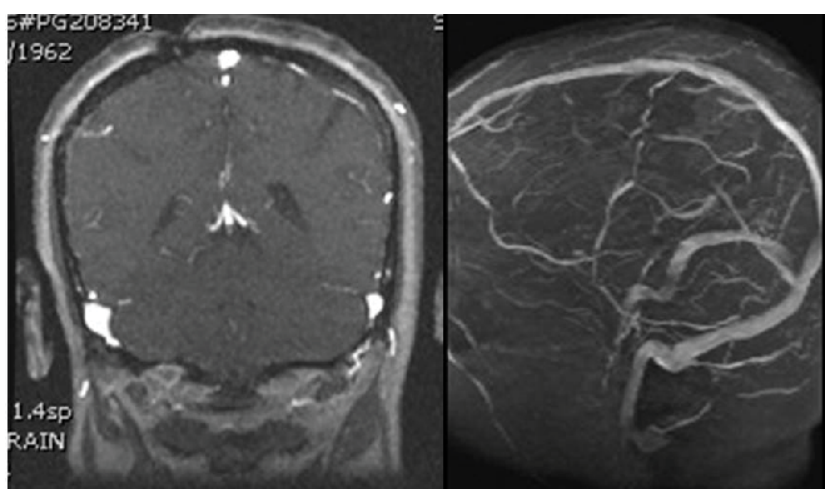

Figure 3: Post-operative brain angio-MR showing total removal of the meningioma and complete patency of the SSS.

leaks [1]. A lot of similar systems which serve the same purpose are commercially available, even if each of them is characterized by peculiar features. The use of autologous fibrin glue obtained directly from the patient's peripheral blood, has been recently described in the neurosurgical settings as a dural sealant [2] but has never been reported to be used as an hemostatic agent. In the case hereby reported, we used autologous fibrin glue as an hemostatic tool in an emergency intraoperative neurosurgical complication. It does not need separate thrombin components, so it is easily and quickly available. Unlike other similar devices, this is the only one with exclusively autologous elements, it does not contain any animal or human homologous components, and it is aprotinin and tranexamic acid-free, elements that are usually added to other fibrin sealant to delay the degradation of the fibrin clot. Therefore, it is neither immunogenic, nor neurotoxic, and a well-tolerated and biocompatible device. Among the different ways of utilization of fibrin glue, only in a few cases it has been used to repair venous sinuses. Up to now, there are no previous reports, from the available literature, concerning the utility of fibrin glue as a haemostatic and reparative agent after surgical injury of the SSS, and the technique to achieve haemostasis and sinus reparation is currently not standardized $[3,4]$. The advent of stereotactic radiosurgery drastically reduced the incidence of iatrogenic rupture of the SSS, restricting the indications for radical Simpson I microsurgical removal of parasagittal meningiomas, particularly with reference to type II, III, IV and V according to Sindou's classification [5]. However, there is a lot of controversy about the utility and possible contraindications to stereotactic radiosurgery for the treatment of parasagittal meningiomas, since it does not always seem efficacious in tumor growth control in long- term outcome, and could determine sinus occlusion through a mechanism of endothelial proliferation $[5,6]$.

As a result, a more aggressive surgical attitude leads to important considerations concerning the modalities to obtain haemostasis and, at the same time, on occurrence, repair the SSS. This is the first report of the available literature, concerning autologous fibrin glue as a haemostatic and reparative tool in parasagittal meningioma surgery. According to our experience, the injection of autologous fibrin glue represents a safe and efficacious technique to stop the bleeding from the damaged SSS rapidly, to guarantee venous repair with satisfactory blood flow restoration, and also to prevent thrombosis and venous infarction.

\section{References}

1. Sekhar LN, Natarajan SK, Manning T, Bhgawati BA (2007) The use of fibrin glue to stop venous bleeding in the epidural space, vertebral venous plexus, and anterior cavernous sinus: technical note. Neurosurgery 61: 51.

2. Giugno A, Maugeri R, D’Arpa S, Visocchi M, lacopino DG (2014) Complex reconstructive surgery following removal of extra-intracranial meningiomas including the use of autologous fibrin glue and a pedicled muscle flap. Interdisciplinary Neurosurgery: Advanced Techniques and Case Management 1: 84-87.

3. Bonnal J, Brotchi J (1978) Surgery of the superior sagittal sinus in parasagittal meningiomas. J. Neurosurgery 48: 938-945

4. Hoessly GF, Olivecrona H (1955) Report on 280 cases of verified parasagittal meningioma. J Neurosurg 12: 614-626.

5. Sindou MP, Alvernia JE (2006) Results of attempted radical tumor remova and venous repair in 100 consecutive meningiomas involving the major dural sinuses. J Neurosurg 105: 514-525

6. Raza SM, Gallia GL, Brem H, Weingart JD, Long DM, et al. (2010) Perioperative and long-term outcomes from the management of parasagittal meningiomas invading the Superior Sagittal Sinus. Neurosurge 7: 885-893. 